\title{
Subtropical Forages Differentially Influenced the Ruminal Fermentation and Microbial Community of Jersey Cow In Vitro
}

\author{
Muhammad Wasim Iqbal ${ }^{1 *}$, Qin Zhang ${ }^{1}$, Caixia Zou ${ }^{1}$, Yingbai Yang ${ }^{1}$, Chunhua Huang ${ }^{2}$ and Bo Lin ${ }^{1}$ \\ ${ }^{1}$ College of Animal Science and Technology, Guangxi University, Nanning, China \\ ${ }^{2}$ Guangxi Institute of Animal Husbandry, Nanning, China
}

"Corresponding author: Iqbal MW, College of Animal Science and Technology, Guangxi University, Nanning, China, Tel: +861878995352; E-mail: linbo@gxu.edu.cn

Received date: August 14, 2018; Accepted date: August 31, 2018; Published date: September 10, 2018

Copyright: @2018 lqbal MW, et al. This is an open-access article distributed under the terms of the Creative Commons Attribution License, which permits unrestricted use, distribution, and reproduction in any medium, provided the original author and source are credited.

\begin{abstract}
Oven dried cassava residues, corn straw silage, elephant grass and sugarcane tail silage were used as substrates to do a $24 \mathrm{~h}$ of incubation with a $100 \mathrm{ml}$ glass syringe at $39^{\circ} \mathrm{C}$. Gas production was recorded at the end of incubation and ruminal fluid was harvested to determine volatile fatty acids (VFA) using gas chromatograph, quantify microbial populations using real time PCR, and analyze microbial community using high throughput sequencing. Results showed in vitro incubation not only decreased population of bacteria, fungi, methanogen and some cellulolytic bacteria $(P<0.05)$, but also increased diversity of bacteria, reversed Firmicutes to Bacteroidetes ratio, and decreased abundance of Prevotella, M. gottschalkii and Entodinium. Gas production, acetate/propionate ratio and abundances of Succiniclasticum, Entodinium and Diploplastron were the highest, while total VFA concentration, fungal and cellulolytic bacterial populations, and abundances of Methanomassiliicoccales and Ostracodinium were the lowest with cassava residues $(P<0.05)$. Influence on fermentation pattern and microbiota of three gramineous substrates was similar, but inoculum incubated with sugarcane tail silage had higher abundance of Methanomassiliicoccales and Diplodinium. In conclusion, cassava residues which is a low neutral detergent fiber forage showed a completely different fermentation pattern and influence on microbe community indicated NDF was the most crucial factor to determine microbial community in vitro.
\end{abstract}

Keywords: Subtropical forages; In vitro fermentation; Microbial community; Microbial population

\section{Introduction}

Forage usually makes up half or more of the ruminants' diet, influencing dry matter intake and microbial community composition both in vivo and in vitro [1-3]. On account of distinct nutritive profile, different forages have discrete impacts on ruminal fermentation and microbiota [4,5]. Changes in the ruminal microbial community induced by forage can provide a clear understanding of interaction between forage and microbes [6]. Due to high biodiversity in tropical and subtropical areas, a variety of roughages is available for the ruminants. However, sustainably available roughage sources used for the ruminants on commercial scale are still the by-products of agricultural cultivations. Among those, cassava starch residues, corn straw silage, sugarcane tail silage and elephant grass are the typical representatives and widely used in the southern China. Cassava starch residue is a source of non-forage fiber which has potential to be used as both beef and dairy cattle diet, as its dry matter contains low fiber but high soluble carbohydrates [7]. Corn straw is a by-product of edible corn produced in the subtropical areas and intensively used as forage [8]. Sugarcane is the most productive crop in the tropical areas, and its tail that contains most of leaves is a nutritious forage for the ruminants [9]. Elephant grass is a fast-growing plant and famous for its higher production in the subtropical areas. It is extensively used as a stable forage source for the ruminants [10].

The microbes inhabiting the gut/rumen are known to impose protective effects and nutritional benefits to the host [11] and due to their superior metabolic potentials compared to the host they are rightly considered equivalent to an organ $[12,13]$. Composition of the microbial community in the rumen and the end products of fermentation depend on the diet fed to the animals [14,15]. To study the impacts of forage source on ruminal fermentation, the in vitro techniques are widely used to realize more controlled and reproducible conditions, compared to in vivo experiments [16]. On the other hand, the advancement in latest microbial molecular techniques, in particular, high throughput sequencing technology have enabled to explore the rumen microbial consortium with higher precision.

Although, nutritional values and digestibility of these typical subtropical forages have been highly explored previously in both in vitro and in vivo studies, but their differential impacts on the ruminal microbial community are poorly researched due to limitations of microbial molecular research techniques in the past. Thus, the primary objective of this study was to examine the impacts of four typical subtropical forages on the ruminal fermentation, microbial population and community composition. Secondly, this study also compared the changes in microbial community composition before and after the incubation to elucidate the effect of fiber structure on ruminal microbiota and fermentation.

\section{Materials and Methods}

\section{Ethical statement}

Ruminal fluid inoculum donors Jersey cows in this study were housed at the Buffalo Research Institute, Chinese Academy of Agricultural Sciences, Nanning, Guangxi province, China. All the experimental protocols regarding animal handling and treatment were approved by the Animal Care Committee, Guangxi University, under 
guidance of the International Cooperation Committee of Animal Welfare, China.

\section{Rumen inoculum donors and their rations}

Rumen inoculum was collected from three ruminal cannulated dry pregnant Jersey cows (Bos taurus) with similar live weights ( $500 \mathrm{~kg})$, before their morning feeding. Animals received $3 \mathrm{~kg}$ concentrate per head per day and corn silage for ad libitum, and free access. Composition of the concentrate feed offered to the animals was as follows (based on dry matter): maize $52 \%$, wheat bran $18.5 \%$, soybean meal $8 \%$, cotton seed meal $15 \%$, stone dust $2 \%$, calcium hydrogen phosphate $1.5 \%$, sodium chloride $2 \%$ and premix $1 \%$. The premix contained per kilogram: $11.9 \mathrm{~g}$ of $\mathrm{MgSO}_{4} \cdot \mathrm{H}_{2} \mathrm{O}, 2.5 \mathrm{~g}$ of $\mathrm{FeSO}_{4} \cdot 7 \mathrm{H}_{2} \mathrm{O}$, $0.8 \mathrm{~g}$ of $\mathrm{CuSO}_{4} \cdot 5 \mathrm{H}_{2} \mathrm{O}, 3 \mathrm{~g}$ of $\mathrm{MnSO}_{4} \cdot \mathrm{H}_{2} \mathrm{O}, 5 \mathrm{~g}$ of $\mathrm{ZnSO}_{4} \cdot \mathrm{H}_{2} \mathrm{O}, 10 \mathrm{mg}$ of $\mathrm{Na}_{2} \mathrm{SeO}_{3}, 40 \mathrm{mg}$ of KI, $30 \mathrm{mg}$ of $\mathrm{CoCl}_{2} \cdot 6 \mathrm{H}_{2} \mathrm{O}, 28.5 \mathrm{~g}$ of vitamin $\mathrm{A} 1$, $0.44 \mathrm{~g}$ of vitamin $\mathrm{D}$, and $16.2 \mathrm{~g}$ of vitamin $\mathrm{E}$.

\section{Substrates and their nutritional composition analysis}

Cassava residues (Manihot esculenta), corn straw silage (Zea mays), elephant grass (Pennisetum purpureum), and sugarcane tail silage (Saccharum officinarum) were used as substrates for in vitro fermentation, and taken from the farm of Buffalo Research Institute, Chinese Academy of Agricultural Sciences, Nanning, Guangxi province, China. Forage samples were dried at $65^{\circ} \mathrm{C}$, ground through 1 millimeter $(\mathrm{mm})$ screen and stored at $-20^{\circ} \mathrm{C}$ until analysed for nutritional composition and in vitro gas production. Forages samples were analysed for dry matter (DM) contents by oven-drying for 8 hours (h) at $105^{\circ} \mathrm{C}$, and crude protein $(\mathrm{CP})$ was calculated as $\mathrm{N} \times 6.25$ [17]. Neutral detergent fibre (NDF) and acid detergent fibre (ADF) contents were determined according to the method as described by Van Soest et al. [18]. Nutritional composition of the four substrates is shown in Table 1.

\begin{tabular}{|l|l|l|l|l|}
\hline \multirow{2}{*}{ Nutrients } & \multicolumn{4}{|c|}{ Substrates } \\
\cline { 2 - 5 } & $\begin{array}{l}\text { Cassava } \\
\text { residues }\end{array}$ & $\begin{array}{l}\text { Corn straw } \\
\text { silage }\end{array}$ & $\begin{array}{l}\text { Elephant } \\
\text { grass }\end{array}$ & $\begin{array}{l}\text { Sugarcane tail } \\
\text { silage }\end{array}$ \\
\hline Dry matter\% & 95.8 & 95.3 & 93.6 & 95 \\
\hline Protein\% & 2.26 & 10.8 & 13.7 & 7.68 \\
\hline $\begin{array}{l}\text { Neutral } \\
\text { detergent fiber } \\
\%\end{array}$ & 21.5 & 52.4 & 57.9 & 68.3 \\
\hline $\begin{array}{l}\text { Acid detergent } \\
\text { fiber\% }\end{array}$ & 15.7 & 30.9 & 32.5 & 37.6 \\
\hline
\end{tabular}

Table 1: Nutritive values of substrates used for in vitro incubation (Dry matter basis).

\section{In vitro fermentation and gas production}

In vitro fermentation system was set up following the procedure as described by Tang et al. [19]. Equal volumes of rumen inoculum taken from the selected three Jersey cows were mixed together. Rumen contents were strained through a four layered cheesecloth into a prewarmed Erlenmeyer flask. All the laboratory handling of rumen inoculum was performed under the continuous flow of carbon dioxide $\left(\mathrm{CO}_{2}\right)$ gas. In vitro fermentation process was carried out in glass syringes $(100 \mathrm{ml})$ fitted with plungers [19]. Every glass syringe was anaerobically dispensed with fermentation medium comprising: $10 \mathrm{ml}$ of rumen inoculum, $20 \mathrm{ml}$ of McDougall's buffer solution and $200 \mathrm{mg}$ of dried forage as substrate. For every substrate, eight experimental replicates $(n=8)$ were set devised and resulting 32 in total. In addition, a similar set of four glass syringes containing only fermentation medium was also run to serve as the blank controls to correct the gas production resulting of fermentation of dry matter in the rumen inoculum. Every glass syringe individually containing fermentation medium and substrate was incubated in a shaking water bath at $39^{\circ} \mathrm{C}$ and gas production was recorded after $24 \mathrm{hr}$ of incubation.

\section{Sampling and volatile fatty acids analysis}

After $24 \mathrm{hr}$ of incubation, fermentation process in four of the eight replicates was ceased by placing them into an ice-cold water bath, and samples of fermented rumen inoculum were collected immediately. Collected samples were filtered through a four layered cheese cloth into a $50 \mathrm{ml}$ centrifuge tube. A $2 \mathrm{ml}$ aliquot of the filtrate was instantly subjected to determining the concentrations of volatile fatty acids (VFA) by using a gas chromatograph (GC-2010, Shimadzu, Tokyo, Japan), equipped with a flame ionization detector and a capillary column (HP-INNOWAX, 1909N-133, Agilent Technologies, Santa Clara, CA, USA) as described by Zhang et al. [20]. Another $2 \mathrm{ml}$ aliquot of the filtrate was stored at $-20^{\circ} \mathrm{C}$ for metagenomic DNA extraction.

\section{DNA extraction and real time quantitative PCR (qRT-PCR)}

DNA was extracted from $2 \mathrm{ml}$ of the preserved sample following the procedure as reported by Rius et al. [21], and further employed to perform quantitative real-time PCR (qRT-PCR) to quantify the populations of bacteria, methanogen, fungi, protozoa, Ruminococcus albus, Fibrobacter succinogenes, Selenomonas ruminantium, and Prevotella ruminicola [22]. Primers used were the same as described by Jiao et al. (Table 2) [22]. Briefly; standard curves were generated by tenfold serial dilutions of plasmid DNAs containing the extracts of $16 \mathrm{~s}$ and 18s rRNA gene inserts from every microbial group and bacterial species. qRT-PCR assay was performed with a $10 \mu \mathrm{L}$ reaction mixture volume using SYBR Green Master Mix (Perfect Real Time Takara, Japan), on a Roche light cycle 480 real time PCR system (Riche, Basel, Switzerland). Reaction mixture contained $5 \mu \mathrm{L}$ of Fast SYBR Green Master Mix, $0.5 \mu \mathrm{L}$ of each primer $\left(20 \mathrm{pmol} \mu \mathrm{L}^{-1}\right), 3.5 \mu \mathrm{L}$ of nucleasefree water and $0.5 \mu \mathrm{L}$ of DNA template $\left(10 \mathrm{ng} \mu \mathrm{L}^{-1}\right)$. All standard dilutions and samples were assayed in triplicate with amplification carried out according to the following program: $95^{\circ} \mathrm{C}$ for $10 \mathrm{~min}$ for initial denaturation, then 30 cycles at $95^{\circ} \mathrm{C}$ for $20 \mathrm{~s}$, annealing for $30 \mathrm{~s}$ at $62^{\circ} \mathrm{C}$, followed by terminal elongation at $72^{\circ} \mathrm{C}$ for $5 \mathrm{~min}$. The corresponding qRT-PCR efficiency for every microbial group and bacterial species ranged from $90 \%$ to $100 \%$. Total $16 \mathrm{~S}$ rRNA and $18 \mathrm{~S}$ rRNA gene copy numbers in samples were determined by relating the threshold cycle values to the standard curves. Copy numbers for the $16 \mathrm{~S}$ rRNA gene in $\mathrm{ml}$ of rumen inoculum were calculated as proposed by Li et al. [23]. Values were converted to log 10 for further statistical analysis.

\begin{tabular}{|c|c|c|c|}
\hline Primer's name & Sequence (5'-3') & $\begin{array}{l}\text { Siz } \\
\text { e } \\
\text { (bp) }\end{array}$ & $\begin{array}{l}\text { Literature } \\
\text { cited }\end{array}$ \\
\hline Bacteria-F & CGGCAACGAGCGCAACCC & & Denman \\
\hline Bacteria-R & CCATTGTAGCACGTGTGTAGCC & 146 & McSweeney \\
\hline
\end{tabular}


Citation: Iqbal MW, Zhang Q, Zou C, Yang Y, Huang C, et al. (2018) Subtropical Forages Differentially Influenced the Ruminal Fermentation and Microbial Community of Jersey Cow In Vitro. J Vet Sci Technol 9: 558. doi:10.4172/2157-7579.1000558

Page 3 of 8

\begin{tabular}{|c|c|c|c|}
\hline Fungi-F & $\begin{array}{l}\text { GAGGAAGTAAAAGTCGTAACAAGGTT } \\
\text { TC }\end{array}$ & \multirow[b]{2}{*}{120} & \multirow{2}{*}{$\begin{array}{l}\text { Denman } \\
\text { and } \\
\text { McSweeney }\end{array}$} \\
\hline Fungi-R & CAAATTCACAAAGGGTAGGATGATT & & \\
\hline Protozoa-F & GCTTTCGWTGGTAGTGTATT & \multirow[b]{2}{*}{223} & \multirow[b]{2}{*}{ Sylvester } \\
\hline Protozoa-R & СTTGCССTCYAATCGTWCT & & \\
\hline Methanogen-F & TTCGGTGGATCDCARAGRGC & \multirow[b]{2}{*}{140} & \multirow[b]{2}{*}{ Denman } \\
\hline Methanogen-R & GBARGTCGWAWCCGTAGAATCC & & \\
\hline $\begin{array}{l}\text { Fibrobacter } \\
\text { succinogenes-F }\end{array}$ & GTTCGGAATTACTGGGCGTAAA & & \multirow{2}{*}{$\begin{array}{l}\text { Denman } \\
\text { and } \\
\text { McSweeney }\end{array}$} \\
\hline $\begin{array}{l}\text { Fibrobacter } \\
\text { succinogenes-R }\end{array}$ & CGCCTGCCCCTGAACTATC & 121 & \\
\hline $\begin{array}{l}\text { Selenomonas } \\
\text { ruminantium- } \mathrm{F}\end{array}$ & CAATAAGCATTCCGCCTGGG & & \multirow[b]{2}{*}{$\begin{array}{l}\text { Stevenson } \\
\text { and Weimer }\end{array}$} \\
\hline $\begin{array}{l}\text { Selenomonas } \\
\text { ruminantium-R }\end{array}$ & TTCACTCAATGTCAAGCCCTGG & 138 & \\
\hline $\begin{array}{l}\text { Ruminococcus } \\
\text { albus-F }\end{array}$ & CCCTAAAAGCAGTCTTAGTTCG & & \multirow[b]{2}{*}{$\begin{array}{l}\text { Koike and } \\
\text { Kobayashi }\end{array}$} \\
\hline $\begin{array}{l}\text { Ruminococcus } \\
\text { albus-R }\end{array}$ & CCTCCTTGCGGTTAGAACA & 176 & \\
\hline $\begin{array}{l}\text { Prevotella } \\
\text { ruminicola-F }\end{array}$ & GAAAGTCGGATTAATGCTCTATGTTG & \multirow[b]{2}{*}{74} & \multirow[b]{2}{*}{ Stevenson } \\
\hline $\begin{array}{l}\text { Prevotella } \\
\text { ruminicola-R }\end{array}$ & CATCCTATAGCGGTAAACCTTTGG & & \\
\hline
\end{tabular}

Table 2: Primers used for qRT-PCR.

\section{High throughput sequencing and bioinformatics analysis}

Four DNA samples from every substrate were pooled into one sample to analyze microbial community. Metagenomic DNA samples were sent to the BGI genomic research center in Wuhan, China, for ruminal microbial community composition analysis. High throughput sequencing technique was conducted using illumina Miseq PE 250 platform (Illumina, Santiago, CA, USA). Bacterial and methanogen communities were analyzed using 16S rRNA gene sequencing, while protozoal community was analyzed using $18 \mathrm{~S}$ rRNA gene sequencing [24]. Primers used for PCR amplifications are shown in Table 3. Sequence data reported in this study have been deposited in the NCBI database (accession no. SRR5930258-SRR5930269). All data processing including sequence quality control, operational taxonomic unit (OTU) based analysis, taxonomy analysis and diversity indices calculations, were performed using the Mothur V 1.31.2 [25]. Bacterial, methanogen and protozoal sequences were grouped into OTUs sharing 97\% similarity. Bacterial 16S rRNA genes were blasted against the Green genes database [26] and methanogen 16S rRNA genes against databases provided by Seedorf [27], while protozoal 18S rRNA genes were blasted against databases provided by Kittelmann and Janssen [28]. Bacterial data were summarized at phylum and genus levels and protozoal data were summarized at genus level, while methanogens data were summarized using a mixed taxonomic rank scheme [29]. Microbial taxa those represented $>1 \%$ of the total community within every microbial group (bacteria, methanogen, and protozoa) were included in the analysis.

\begin{tabular}{|l|l|l|l|}
\hline Microbes & Primer sequence $\mathbf{( 5}^{\prime} \mathbf{3}^{\prime} \mathbf{)}$ & $\begin{array}{l}\text { Size } \\
\mathbf{( b p )}\end{array}$ & $\begin{array}{l}\text { Literature } \\
\text { cited }\end{array}$ \\
\hline Bacteria-F & GGCGVACGGGTGAGTAA & 427 & Hristov \\
\hline Bacteria-R & CCGCNGCNGCTGGCAC & & \\
\hline Methanogen-F & GCGGTGTGTGCAAGGAGC & \multirow{2}{*}{472} & $\mathrm{Jin}$ \\
\hline Methanogen-R & AGGAATTGGCGGGGGAGCAC & & \\
\hline Protozoa-F & AATTGCAAAGATCTATCCC & & Kittelmann \\
\hline Protozoa-R & GACTAGGGATTGGAGTGG & & \\
\hline
\end{tabular}

Table 3: Primers used for microbial community composition analysis.

\section{Statistical analysis of data}

All the preliminary data including rumen fermentation parameters, microbial population and microbial relative abundance were sorted by Microsoft excel. Ruminal fermentation and microbial population data were analyzed as a one-way factorial design using the ANOVA procedure of SAS (2005), according to the following statistical model:

$$
\mathrm{Y}_{\mathrm{i}}=\mu+\alpha_{\mathrm{i}}+\varepsilon_{\mathrm{i}}
$$

Where: $Y_{i}$ is dependent variable, $\alpha_{i}$ is effect of substrate $(i=1,4)$ and $\varepsilon_{\mathrm{i}}$ is the residual error. Differences among means were tested using Duncan's multiple range tests. Statistical significance was considered if $\mathrm{P}<0.05$.

\section{Results}

\section{Effects of substrate on in vitro gas production, fermentation parameters and microbial population}

After $24 \mathrm{~h}$ of incubation, the highest GP, butyrate concentration and acetate/propionate (A/P) ratio, while the lowest total VFA, acetate and propionate concentrations were observed on incubation with cassava residues $(\mathrm{P}<0.05$, Table 4$)$. Second highest GP, the highest propionate concentration and the lowest $\mathrm{A} / \mathrm{P}$ ratio resulted with corn straw silage among the four substrates $(\mathrm{P}<0.05)$. The lowest $\mathrm{GP}$ and butyrate $(\mathrm{P}<0.05)$, while the highest total VFA and acetate concentrations $(\mathrm{P}>0.05)$ were observed with elephant grass. Acetate, propionate as well as total VFA concentrations after incubation with the four substrates were lower than those in fresh rumen inoculum. For the microbial populations; it was found the gene number of bacteria, fungi, $P$. ruminicola, $R$. albus, $F$ succinogenes, and $S$. ruminantium were lower with cassava residues $(\mathrm{P}<0.05$, Table 4$)$, while higher with elephant grass $(\mathrm{P}<0.05)$. The highest gene numbers of protozoa and methanogens were observed with cassava residues $(\mathrm{P}<0.05)$. Except protozoal population was increased with cassava residues, populations of all the other microbes were decreased as compared with those in fresh rumen inoculum.

\begin{tabular}{|l|l|l|l|l|l|l|}
\hline \multirow{2}{*}{} & \multicolumn{4}{|c|}{ Substrate source } & $\begin{array}{l}\text { SE } \\
\text { M }\end{array}$ & $\mathbf{P}$ \\
\cline { 3 - 6 } & & & $\begin{array}{l}\text { Cor } \\
\text { n }\end{array}$ & & \\
Index & $\begin{array}{l}\text { Fresh } \\
\text { rumen } \\
\text { inoculu } \\
\text { m }\end{array}$ & $\begin{array}{l}\text { Cassa } \\
\text { va } \\
\text { residu } \\
\text { es }\end{array}$ & $\begin{array}{l}\text { stra } \\
\text { w } \\
\text { silag } \\
\text { es }\end{array}$ & $\begin{array}{l}\text { Elepha } \\
\text { nt } \\
\text { grass }\end{array}$ & $\begin{array}{l}\text { Sugarca } \\
\text { ne tail } \\
\text { silage }\end{array}$ & \\
\hline
\end{tabular}


Citation: Iqbal MW, Zhang Q, Zou C, Yang Y, Huang C, et al. (2018) Subtropical Forages Differentially Influenced the Ruminal Fermentation and Microbial Community of Jersey Cow In Vitro. J Vet Sci Technol 9: 558. doi:10.4172/2157-7579.1000558

Page 4 of 8

\begin{tabular}{|l|l|l|l|l|l|l|l|}
\hline $\begin{array}{l}\text { Gas production, } \\
\text { (mL/g) }\end{array}$ & - & $354 \mathrm{~A}$ & $\begin{array}{l}171 \\
\mathrm{~B}\end{array}$ & $151 \mathrm{C}$ & $166 \mathrm{~B}$ & $\begin{array}{l}3.8 \\
8\end{array}$ & 0.02 \\
\hline TVFA, (mmol/L) & $74.7 \mathrm{~A}$ & $60.3 \mathrm{C}$ & $\begin{array}{l}64.4 \\
\mathrm{~B}\end{array}$ & $65.0 \mathrm{~B}$ & $62.8 \mathrm{~B}$ & $\begin{array}{l}4.5 \\
8\end{array}$ & 0.03 \\
\hline $\begin{array}{l}\text { Acetate, } \\
\text { (mmol/L) }\end{array}$ & $54.8 \mathrm{~A}$ & $42.0 \mathrm{C}$ & $\begin{array}{l}45.6 \\
\mathrm{~B}\end{array}$ & $48.7 \mathrm{~B}$ & $45.0 \mathrm{~B}$ & $\begin{array}{l}3.0 \\
9\end{array}$ & 0.01 \\
\hline $\begin{array}{l}\text { Propionate, } \\
\text { (mmol/L) }\end{array}$ & $10.1 \mathrm{~A}$ & $7.85 \mathrm{~B}$ & $\begin{array}{l}10.6 \\
\mathrm{~A}\end{array}$ & $9.51 \mathrm{~A}$ & $9.62 \mathrm{~A}$ & $\begin{array}{l}0.9 \\
3\end{array}$ & 0.04 \\
\hline $\begin{array}{l}\text { Butyrate, } \\
\text { (mmol/L) }\end{array}$ & $9.81 \mathrm{~A}$ & $10.4 \mathrm{~A}$ & $\begin{array}{l}8.25 \\
\mathrm{~B}\end{array}$ & $6.82 \mathrm{C}$ & $8.26 \mathrm{~B}$ & $\begin{array}{l}0.6 \\
7\end{array}$ & 0.01 \\
\hline $\begin{array}{l}\text { Acetate/ } \\
\text { Propionate }\end{array}$ & $5.46 \mathrm{~A}$ & $5.37 \mathrm{~A}$ & $\begin{array}{l}4.29 \\
\mathrm{~B}\end{array}$ & $5.12 \mathrm{~A}$ & $4.68 \mathrm{~A}$ & $\begin{array}{l}0.2 \\
3\end{array}$ & 0.01 \\
\hline $\begin{array}{l}\text { Bacteria, } \\
\text { log10(copy/mL) }\end{array}$ & 9.95 & 9.13 & 9.36 & 9.46 & 9.39 & $\begin{array}{l}0.0 \\
8\end{array}$ & $<0.0$ \\
\hline $\begin{array}{l}\text { Methanogen, } \\
\text { log10(copy/mL) }\end{array}$ & 8.12 & 7.41 & 7.06 & 7.22 & 7.15 & $\begin{array}{l}0.2 \\
4\end{array}$ & 0.01 \\
\hline $\begin{array}{l}\text { Fungi, } \\
\text { log10(copy/ml) }\end{array}$ & 5.88 & 4.4 & 5.61 & 6.51 & 6.36 & $\begin{array}{l}0.2 \\
7\end{array}$ & $\begin{array}{l}<0.0 \\
1\end{array}$ \\
\hline $\begin{array}{l}\text { Protozoa, } \\
\text { log10(copy/ml) }\end{array}$ & 7.35 & 7.65 & 7.12 & 6.94 & 7.13 & $\begin{array}{l}0.2 \\
1\end{array}$ & 0.02 \\
\hline $\begin{array}{l}\text { R. albus, } \\
\text { log10(copy/ml) }\end{array}$ & 6.7 & 5.58 & 6.29 & 6.46 & 6 & $\begin{array}{l}0.1 \\
5\end{array}$ & $\begin{array}{l}<0.0 \\
1\end{array}$ \\
\hline $\begin{array}{l}\text { F succinogenes } \\
\text { log10(copy/mL) }\end{array}$ & 6.97 & 5.47 & 6.23 & 6.58 & 6.44 & $\begin{array}{l}0.1 \\
2\end{array}$ & $\begin{array}{l}<0.0 \\
1\end{array}$ \\
\hline $\begin{array}{l}\text { P. ruminicola, } \\
\text { log10(copy/mL) }\end{array}$ & 8.81 & 7 & 7.89 & 8.19 & 8.13 & 0.2 & 0.01 \\
\hline $\begin{array}{l}\text { S. ruminantium, } \\
\text { log10(copy/mL) }\end{array}$ & 8.71 & 8.06 & 8.42 & 8.52 & 8.45 & $\begin{array}{l}0.1 \\
7\end{array}$ & 0.01 \\
\hline
\end{tabular}

\begin{tabular}{|c|c|c|c|c|c|c|}
\hline \multirow[b]{2}{*}{$\begin{array}{l}\text { Rumen } \\
\text { microbes }\end{array}$} & \multirow[b]{2}{*}{ Index } & \multicolumn{5}{|c|}{ Substrate source } \\
\hline & & $\begin{array}{l}\text { Fresh } \\
\text { rumen } \\
\text { inoculu } \\
\text { m }\end{array}$ & $\begin{array}{l}\text { Cassav } \\
\text { a } \\
\text { residue } \\
\mathbf{s}\end{array}$ & $\begin{array}{l}\text { Corn } \\
\text { straw } \\
\text { silag } \\
\text { e }\end{array}$ & $\begin{array}{l}\text { Elephan } \\
t \text { grass }\end{array}$ & $\begin{array}{l}\text { Sugarcan } \\
\text { e tail } \\
\text { silage }\end{array}$ \\
\hline \multirow[t]{3}{*}{ Bacteria } & Chao1 & 706 & 787 & 876 & 920 & 960 \\
\hline & Shannon & 4.94 & 5.13 & 5.59 & 5.68 & 5.71 \\
\hline & Simpson & 0.02 & 0.02 & 0.01 & 0.01 & 0.01 \\
\hline \multirow[t]{3}{*}{ Methanogen } & Chao1 & 11.5 & 9 & 11 & 16 & 11 \\
\hline & Shannon & 0.53 & 1.28 & 1.21 & 1.36 & 1.26 \\
\hline & Simpson & 0.78 & 0.32 & 0.37 & 0.29 & 0.33 \\
\hline \multirow[t]{3}{*}{ Protozoa } & Chao1 & 161 & 162 & 323 & 238 & 315 \\
\hline & Shannon & 3.28 & 2.01 & 3.44 & 3.16 & 3.64 \\
\hline & Simpson & 0.07 & 0.36 & 0.09 & 0.12 & 0.06 \\
\hline
\end{tabular}

Table 5: Ruminal microbial Alpha diversity statistics after $24 \mathrm{hr}$ of in vitro incubation with four different substrates.

\begin{tabular}{|c|c|c|c|c|c|}
\hline \multirow[b]{2}{*}{ Taxon } & \multicolumn{5}{|c|}{ Substrate source } \\
\hline & $\begin{array}{l}\text { Fresh } \\
\text { rumen } \\
\text { inoculum }\end{array}$ & $\begin{array}{l}\text { Cassava } \\
\text { residues }\end{array}$ & $\begin{array}{l}\text { Corn } \\
\text { straw } \\
\text { silage }\end{array}$ & $\begin{array}{l}\text { Elephant } \\
\text { grass }\end{array}$ & $\begin{array}{l}\text { Sugarcane } \\
\text { tail silage }\end{array}$ \\
\hline \multicolumn{6}{|l|}{ Phylum } \\
\hline Firmicutes & 35.3 & 55.5 & 55.9 & 54.3 & 52.2 \\
\hline Bacteroidetes & 60.1 & 35.4 & 36.4 & 37 & 41.4 \\
\hline Proteobacteria & 0.24 & 0.92 & 3.67 & 4.4 & 2.49 \\
\hline Chloroflexi & 1.19 & 6.68 & 2.09 & 1.56 & 1.87 \\
\hline Fibrobacteres & 0.01 & 0 & 0.09 & 0.15 & 0.12 \\
\hline Spirochaetes & 0.83 & 0.38 & 0.67 & 0.95 & 0.68 \\
\hline Synergistetes & 0.01 & 0.09 & 0.21 & 0.2 & 0.22 \\
\hline Tenericutes & 0.31 & 0.23 & 0.28 & 0.44 & 0.38 \\
\hline TM7 & 1.11 & 0.62 & 0.5 & 0.74 & 0.38 \\
\hline Unclassified & 0.18 & 0.07 & 0.08 & 0.11 & 0.13 \\
\hline \multicolumn{6}{|l|}{ Genus } \\
\hline Prevotella & 56.5 & 30 & 27.5 & 26.3 & 29 \\
\hline Ruminococcus & 1.15 & 2.94 & 0.86 & 0.93 & 0.74 \\
\hline Succiniclasticum & 1.4 & 7.86 & 1.79 & 1.3 & 1.79 \\
\hline Coprococcus & 1.16 & 0.25 & 1.12 & 2.06 & 1.29 \\
\hline Comamonas & 0 & 0.06 & 2.24 & 3.3 & 1.29 \\
\hline Butyrivibrio & 2.03 & 1.2 & 1.59 & 2.2 & 1.7 \\
\hline Oscillospira & 0.25 & 0.78 & 0.73 & 0.56 & 0.86 \\
\hline Mogibacterium & 0.97 & 0.55 & 0.38 & 0.39 & 0.5 \\
\hline
\end{tabular}

Table 4: Gas production, ruminal fermentation parameters, and microbial populations in inoculum after $24 \mathrm{hr}$ of in vitro incubation with four different substrates. TVFA stands for total volatile fatty acids. Means within a row differ with different superscripts $(\mathrm{P}<0.05)$.

\section{Effects of substrates on ruminal bacterial community}

After $24 \mathrm{hr}$ of incubation, bacterial Chaol and Shannon index were increased, but Simpson index was decreased by in vitro incubation with the four substrates (Table 5). Comparing the four substrates, the lowest bacterial Chaol and Shannon index were observed with cassava residues, there was no difference in diversity indices for the other three substrates (Table 5). Community composition analysis showed, at phylum level, abundances of Firmicutes, Proteobacteria and Chloroflexi were increased, but the abundance of Bacteroidetes was decreased (from $60 \%$ to $<42 \%$ ) by in vitro incubation with the four substrates (Table 6). At genus level, abundance of dominant genus Prevotella was substantially decreased (from $57 \%$ to $<30 \%$ ), while abundance of unclassified bacterial genera was increased (from $33 \%$ to $>46 \%$ ) by in vitro incubation with the four substrates (Table 6). Comparing the four substrates, bacterial community of cassava residues hkad lower abundance of phyla Proteobacteria and Synergistetes, but higher abundance of Chloroflexi than that for other three substrates (Table 6). In addition, cassava residues had higher abundances of bacterial genera Ruminococcus and Succiniclasticum, while lower abundance of unclassified bacterial genera than the other three substrates (Table 6). 
Citation: Iqbal MW, Zhang Q, Zou C, Yang Y, Huang C, et al. (2018) Subtropical Forages Differentially Influenced the Ruminal Fermentation and Microbial Community of Jersey Cow In Vitro. J Vet Sci Technol 9: 558. doi:10.4172/2157-7579.1000558

Page 5 of 8

\begin{tabular}{|l|l|l|l|l|l|}
\hline Clostridium & 0.25 & 0.06 & 0.28 & 0.24 & 0.23 \\
\hline Sphaerochaeta & 0.57 & 0.24 & 0.18 & 0.1 & 0.14 \\
\hline Unclassified & 32.7 & 46.9 & 56.8 & 55.9 & 55.7 \\
\hline Others $(<0.5 \%)$ & 1.67 & 2.35 & 3.87 & 4.33 & 4 \\
\hline
\end{tabular}

Table 6: Bacterial abundance in Jersey cow inoculum at phylum and genus levels after $24 \mathrm{hr}$ of in vitro incubation with four different substrates (percentage of bacteria in total bacteria $>0.5 \%$ ).

\section{Effects of substrates on ruminal methanogen community}

After $24 \mathrm{hr}$ of incubation, methanogen Shannon index was increased but Simpson index was decreased by in vitro incubation with the four substrates (Table 5). Comparing the four substrates, elephant grass had the highest Shannon index and lowest Simpson index as compared with another substrate (Table 5). Community composition analysis showed, the abundance of $M$. gottschalkii which was the dominant methanogen species, was greatly decreased by in vitro incubation with the four substrates (Table 7). The most obvious change observed in methanogen community was increased abundance of Methanomassiliicoccales Group10 sp. substantially after incubation with three gramineous substrates $(>21 \%)$, especially with Sugarcane tail Silage ( $>40 \%$, Table 6$)$; while its abundance in the fresh rumen inoculum was very low $(0.15 \%)$. Comparing the four substrates, methanogen community after incubation with cassava residues appeared with different composition which had higher abundance of Methanobrevibacter gottschalkii, but lower abundance of Methanomassiliicoccales Group10 sp. than that of the other three gramineous substrates (Table 7).

\begin{tabular}{|l|l|l|l|l|l|}
\hline & \multicolumn{5}{|c|}{ Substrate source } \\
\cline { 2 - 6 } & $\begin{array}{l}\text { Fresh } \\
\text { rumen } \\
\text { inoculu } \\
\text { m }\end{array}$ & $\begin{array}{l}\text { Cassav } \\
\text { a } \\
\text { residue } \\
\text { s }\end{array}$ & $\begin{array}{l}\text { Corn } \\
\text { straw } \\
\text { silag } \\
\text { e }\end{array}$ & $\begin{array}{l}\text { Elephan } \\
\text { t grass }\end{array}$ & $\begin{array}{l}\text { Sugarcan } \\
\text { ene } \\
\text { silail }\end{array}$ \\
\hline $\begin{array}{l}\text { Methanobrevibacter } \\
\text { gottschalkii }\end{array}$ & 88.1 & 72.3 & 69.9 & 59.9 & 52.5 \\
\hline $\begin{array}{l}\text { Methanobrevibacter } \\
\text { ruminantium }\end{array}$ & 4.34 & 19.6 & 7.02 & 14.6 & 5.95 \\
\hline $\begin{array}{l}\text { Methanosphaera sp. } \\
\text { Methanomassiliicoccales } \\
\text { Group10 sp. }\end{array}$ & 0.99 & 1.04 & 0.02 & 0.01 & 0.02 \\
\hline $\begin{array}{l}\text { Methanomassiliicoccales } \\
\text { Group12 sp. }\end{array}$ & 0.02 & 0.09 & 22 & 24.2 & 40.1 \\
\hline $\begin{array}{l}\text { Methanobacterium } \\
\text { alkaliphilum }\end{array}$ & 0.05 & 0 & 0.01 & 0.01 & 0.02 \\
\hline Others (<0.5\%) & 0.25 & 0.09 & 0.03 & 0.03 & 0.03 \\
\hline Unclassified & 3.47 & 0.19 & 0.64 & 0.74 & 0.73 \\
\hline
\end{tabular}

Table 7: Methanogen abundance in inoculum at genus level after $24 \mathrm{~h}$ of in vitro incubation with four different substrates. (Percentage of methanogens in total methanogens $>0.5 \%$ ).

\section{Effects of substrates on ruminal protozoal community}

After $24 \mathrm{~h}$ of incubation, protozoal Chaol index was greatly increased by in vitro incubation with three gramineous substrates (from 161 to >238); however, Shannon index was decreased, and Simpson index was increased by in vitro incubation with cassava residues (Table 5). Comparing the four substrates, cassava residues had lower Chaol and Shannon index, but higher Simpson index than those of the other three gramineous substrates (Table 5). Community composition analysis showed, the abundances of Entodinium, Ophryoscolex and Metadinium were decreased, while the abundance of Ostracodinium was increased by in vitro incubation with the four substrates. In particular, abundance of Entodinium was greatly decreased (from $47 \%$ to $<12 \%$ ), while abundance of Ostracodinium was greatly increased (from $1.5 \%$ to $>41 \%$ ) by incubation with three gramineous substrates (Table 8). Besides, abundance of Diploplastron, which was a low abundant genus in fresh rumen inoculum and fermented inocula of the three gramineous substrates $(<3 \%)$, was greatly increased by incubation with cassava residues (from $<9.8 \%$ to $59 \%$ ) Comparing of four substrates, cassava residues had much lower abundance of Ostracodinium, Polyplastron and Ophryoscolex, and much higher abundance of Entodinium and Diploplastron than those of other three gramineous substrates. Sugarcane tail silage had lower abundance of Ostracodinium, but higher abundance of Diplodinium than those of corn straw silage and elephant grass (Table 8).

\begin{tabular}{|l|l|l|l|l|l|}
\hline \multirow{2}{*}{} & \multicolumn{5}{|c|}{ Substrate source } \\
\cline { 2 - 6 } & $\begin{array}{l}\text { Fresh } \\
\text { rumen } \\
\text { inoculum }\end{array}$ & $\begin{array}{l}\text { Cassava } \\
\text { residues }\end{array}$ & $\begin{array}{l}\text { Corn } \\
\text { straw } \\
\text { silage }\end{array}$ & $\begin{array}{l}\text { Elephant } \\
\text { grass }\end{array}$ & $\begin{array}{l}\text { Sugarcane } \\
\text { tail silage }\end{array}$ \\
\hline Ostracodinium & 1.49 & 2.59 & 64.2 & 75.9 & 41.4 \\
\hline Entodinium & 46.7 & 30.3 & 10.8 & 6.76 & 11.1 \\
\hline Polyplastron & 3.58 & 3.22 & 11.4 & 8.24 & 17.7 \\
\hline Ophryoscolex & 19.3 & 1.68 & 9.09 & 5.97 & 5.43 \\
\hline $\begin{array}{l}\text { Anoplodinium.- } \\
\text { Diplodinium }\end{array}$ & 4.93 & 2.04 & 2.29 & 1.66 & 20.1 \\
\hline Metadinium & 10.9 & 0.07 & 0.01 & 0.03 & 0.03 \\
\hline $\begin{array}{l}\text { Employer.- } \\
\text { Diploplastron }\end{array}$ & 9.83 & 59 & 0.89 & 1.12 & 3.35 \\
\hline Eudiplodinium & 1.3 & 1.07 & 0.35 & 0.11 & 0.49 \\
\hline Epidinium & 1.04 & 0 & 0.01 & 0.06 & 0.03 \\
\hline Dasytricha & 0.71 & 0 & 0.01 & 0.08 & 0.01 \\
\hline Unclassified & 0.13 & 0.01 & 0.01 & 0.01 & 0.01 \\
\hline
\end{tabular}

Table 8: Protozoal abundance in inoculum at genus level after $24 \mathrm{~h}$ of in vitro incubation with four different substrates (percentage of protozoa in total protozoa $>0.5 \%$ ).

\section{Discussion}

Standardized method of in vitro batch culture system has been intensively used to evaluate the quality of ruminant's rations recently [30]. Numerous studies have researched the influence of forage source on in vitro gas production and rumen fermentation parameters $[31,32]$. However, studies addressing the changes in ruminal microbial 
community composition after in vitro incubation with different substrates are limited [33]. In contrast, the current study not only focused the effects of substrate on in vitro digestibility of substrates, but it also explored the interaction between forage source and microbial community including bacteria, methanogen and protozoa.

Digestibility of substrates is determined by its in vitro gas production. As cassava residues produced highest gas and VFA, indicting it had the highest in vitro degradability among all the substrates. The potential reason was higher soluble carbohydrate and the lowest NDF contents of cassava residues among the four substrates; because lower NDF content was always related with higher gas production and digestibility in vitro $[34,35]$. The highest butyrate and the lowest acetate concentrations after incubation with cassava residues were probably due to the increased number of protozoa; because butyrate and acetate are two main VFA produced by protozoa on fermentation of starch and cellulose respectively [36-38].

The batch incubation system is characterized for being unable to keep the microbial growth and population steady for long time due to exhaustion of substrates and accumulation of fermentation products. Besides, response of different microbial groups to in vitro incubation was also different which can cause the change in ruminal microbe community profile during in vitro incubation [39]. The population of almost every ruminal microbial group in this study including Bacteria, Methanogen, Fungi, $R$. albus, $F$ succinogenes, $P$. ruminicola and $S$. ruminantium were decreased after in vitro incubation as compared with those in freshly taken ruminal liquor. In particular, fungal population was drastically decreased by incubation with the four substrates. This result was consistent with the findings of Soto et al. [32], who reported that populations of bacteria, fungi, F. succinogenes, $R$. flavefaciens were decreased in fermented inoculum as compared with those in fresh rumen fluid, and this study also reported that bacteria, fungi, methanogens, and F. succinogenes were decreased even though a single-flow continuous-cultivation system was used. The rapid disappearance of fermentable substrates can explain the decrease of these microbial groups, especially cellulolytic bacteria and fungi. Whereas, the probable reason behind the drastic decrease of fungal population was the higher sensitivity of anaerobic fungi to in vitro incubation system.

Due to different nutritional characteristics of substrates, the response of every microbial group to substrates is also different. This can cause alterations in microbial community profile during in vitro incubation [39]. In the present study, in vitro incubation with cassava residues resulted in lower populations of bacteria, fungi, $R$. albus and $F$. succinogenes as compared with those with high fiber containing substrates. These ruminal fiber degrading microbes especially, fungi were decreased in population by incubation with cassava residues which was a low fiber containing substrate. This result indicated low fiber substrate was not beneficial for the growth of fiber degrading microbes during in vitro incubation. This result was also consistent with the findings of Saro et al. [40], who reported abundances of ruminal $F$. succinogenes, $R$. flavefaciens and fungi were higher in high NDF grass hay feeding sheep than in alfalfa hay feeding sheep. Moreover, Huws et al. [41] also reported abundance of ruminal cellulolytic bacteria of steers fed with high NDF grass silage was higher than that fed with low NDF red clover silage.

Firmicutes and Bacteroidetes still stood dominant bacterial phyla after incubation with every substrate. However, abundance of Firmicutes was increased while the abundance of Bcateroidetes was decreased after incubation as compared with those in fresh ruminal fluid; indicating the influence of in vitro incubation on bacterial community. At genus level, Prevotella, a group of multifunctional key microbe in the rumen was greatly decreased in abundance by in vitro incubation with every substrate. Prevotella has been reported to be responsible for cellulose, hemicellulose starch and protein degradation in the rumen $[42,43]$. This suggested that the ability of in vitro system to ferment substrate was depressed as compared with that of in vivo. This result was consistent with reports addressing that ferment ability of in vitro system was lower than that of in vivo [44]. Besides, bacterial diversity was increased by in vitro incubation which was evidenced by increased Shannon diversity index, but the newly increased bacteria were unclassified bacteria in this study. The possible reason was that when high abundant bacteria such as Prevotella were decreased in abundance by in vitro incubation, the less abundant species which were fit for in vitro environment were increased to take the ecological niche. However, Soto et al. [32] reported bacterial diversity was decreased by in vitro batch culture system, which was not consistent with the results of this study. This inconsistent was explained by the difference of method used to explore the diversity. The sequencing method used in this study has higher resolution than terminalrestriction fragment length polymorphism (T-RFLP) which was a method used by Soto et al. [32]; therefore, this study showed higher abundance of unclassified bacterial species than that reported by Soto et al. [32]. Incubation with cassava residues decreased Shannon index, indicating low NDF forages can decrease bacterial diversity. This was consistent with findings of Grilli et al. [45], who reported that goat ruminal bacteria Shannon index was decreased when goat was fed with $60 \%$ alfalfa hay as compared to fed with $100 \%$ alfalfa hay.

Methanogen population was not greatly influenced by in vitro fermentation. However, methanogen community composition was altered, and diversity was increased by incubation with the four substrate. In particular, the abundance of $M$. gottschalkii was decreased, while abundance of Methanomassiliicoccales Group10 sp., previously named as Rumen Cluster C (RCC), was increased after incubation with three gramineous substrates. Methanomassiliicoccales was a group of methanogens that strictly use hydrogen to reduce both methylamines and methanol to methane [46], and degradation products of pectin can promote their growth [47]. Higher abundance of Methanomassiliicoccales in inoculum after incubation with the three gramineous substrates was probably due to higher pectin contents in those substrates, or their fermentation products contained higher methyl compounds; but needs to be further confirmed. RCC are important methane producers in rumen and due to their low abundance, it is hard to get their pure culture. However, in current study we found that in vitro incubation of sugarcane tail silage with Jersey cow rumen fluid can elevate the abundance of Methanomassiliicoccales up to more than $40 \%$, indicating forage with high NDF contents was a better source to get Methanomassiliicoccales enriched.

As compared with methanogen and bacterial, protozoal population and diversities were not greatly influenced by in vitro incubation, while their community demonstrated variations after incubation. Our study found abundance of low abundant protozoa, such as Ostracodinium and Diploplastron, and high abundant Entodinium in fresh rumen liquor were reversed after in vitro incubation with specific substrates. The reason for this change was unclear, but we can predict that this probably be related with inherent variation of protozoal community in ruminants [48], and it has been reported that ruminal protozoal community has a great varation among individual buffalo even though they were feed same diet in same shed. Our study found Entodinium 
which is a dominant protozoal genus in ruminants, took a larger account of protozoal community (31\%) after incubation with cassava residues as compared with the other three gramineous substrates. This result was consistent with findings of Coleman [49], who reported that Entodinium has the highest starch uptake rate as compared with another protozoa group. Thus, higher population of Entodinium with cassava residues was probably due to high starch contents of this substrate. Whereas, the reason for substantially higher abundance of Diploplastron after incubation with cassava residues, and much higher abundance of Ostracodinium after incubation with the three gramineous substrates was still unclear. We can predict that NDF contents of these substrates may be the most probable determiners. Though, three gramineous substrates demonstrated similar fermentation patterns and influence on microbial population and community, but incubation with sugarcane tail silage resulted in higher abundances of Methanomassiliicoccales and Diplodinium than those of the other two gramineous substrates; indicated sugarcane tail silage might have special nutritional characteristics to be evaluated further.

\section{Conclusion}

The $24 \mathrm{hr}$ in vitro batch incubation not only decreased the populations of bacteria, fungi, methanogens and some cellulolytic bacteria, but also changed the microbial community as compared with fresh rumen fluid. The original Firmicutes to Bacteroidetes ratio was reversed and abundance of Prevotella was greatly decreased. Cassava residues which is a low NDF contents substrate greatly decreased fungal and cellulolytic bacterial populations, and increased abundances of Succiniclasticum and Diploplastron as compared with the other three high NDF gramineous substrates. Therefore, it is concluded that NDF contents is important to determine microbial population and community in vitro. Besides, Methanomassiliicoccales was greatly enriched after incubation with sugarcane tail silage indicated in vitro incubation with sepcial substrate was a potential way to enrich special methanogen.

\section{Acknowledgements}

This work was supported by Scientific Research Foundation of Guangxi University (No. XGZ160164) and Guangxi Key Science Research and Development Project (No. GuikeAB6380190).

\section{Conflict of Interest}

We have no conflict of interest.

\section{References}

1. Kong Y, Teather R, Forster R (2010) Composition, spatial distribution, and diversity of the bacterial communities in the rumen of cows fed different forages. FEMS Microbiol Ecol 74: 612-622.

2. Martinez ME, Ranilla MJ, Tejido ML, Saro C, Carro MD (2010) Comparison of fermentation of diets of variable composition and microbial populations in the rumen of sheep and Rusitec fermenters. II. Protozoa population and diversity of bacterial communities. J Dairy Sci 93: 3699-3712.

3. Kendall C, Leonardi C, Hoffman PC, Combs DK (2009) Intake and milk production of cows fed diets that differed in dietary neutral detergent fiber and neutral detergent fiber digestibility. J Dairy Sci 92: 313-323.

4. Brask M, Lund P, Hellwing AL, Poulsen M, Weisbjerg MR (2013) Enteric methane production, digestibility and rumen fermentation in dairy cows fed different forages with and without rapeseed fat supplementation. Animal Feed Sci Technol 184: 67-79.
5. Lettat A, Hassanat F, Benchaar C (2013) Corn silage in dairy cow diets to reduce ruminal methanogenesis: Effects on the rumen metabolically active microbial communities. J Dairy Sci 96: 5237-5248.

6. Yang CT, Bing-Wen SI, Diao QY, Jin H, Zeng SQ, et al. (2016) Rumen fermentation and bacterial communities in weaned Chahaer lambs on diets with different protein levels. J Integr Agric 15: 1564-1574.

7. Bradford BJ, Mullins CR (2012) Invited review: Strategies for promoting productivity and health of dairy cattle by feeding non-forage fiber sources. J Dairy Sci 95: 4735-4746.

8. Cao ZJ, Ma M, Ji P, Li SL (2010) Effects of Maize Silage Particle Size and Feeding Method on Ruminal Fermentation, Chewing Activity and Passage Rate of Lactation Cows. J Animal Vet Adv 9: 374-383.

9. Landell MGA, Campana MP, Rodrigues AA (2002) The variety IAC86-2480 as a new option for sugar cane for fodder purposes: management of production and use in animal feed. Tech Bull IAC193, Technology Series APTA, p: 36.

10. Muia JMK, Tamminga S, Mbugua PN (2000) Effect of supplementing Napier grass (Pennisetum purpureum) with sunflower meal or poultry litter-based concentrates on feed intake, live-weight changes and economics of milk production in Friesian cows. Livest Prod Sci 67: 89-99.

11. Hooper LV (2004) Bacterial contributions to mammalian gut development. Trends Microbiol 12: 129-134.

12. Egert M, De Graaf AA, Smidt H, De Vos WM, Venema K (2005) Beyond diversity: functional microbiomics of the human colon. Trends Microbiol 14: 86-91.

13. Xu J, Gordon JI (2003) Honor thy symbionts. Proc Natl Acad Sci USA 100: 10452-10459.

14. Dewhurst RJ, Wadhwa D, Borgida LP, Fisher WJ (2001) Rumen acid production from dairy feeds. 1. Effects on feed intake and milk production of dairy cows offered grass or corn silages. J Dairy Sci 84: 2721-2729.

15. Belanche A, Doreau M, Edwards JE, Moorby JM, Pinloche E, et al. (2012) Shifts in the rumen microbiota due to the type of carbohydrate and level of protein ingested by dairy cattle are associated with changes in rumen fermentation. J Nutrition 142: 1684-1692.

16. Zapletalova M, Kasparovsks J, Krizova L, Kasparovsky T, Sery O, et al. (2016) Bacterial community dynamics in a rumen fluid bioreactor during in-vitro cultivation. J Biotechnol 234: 43-49.

17. AOAC (1990) Official methods of analysis (15th Edn). Arlington: AOAC International, Rockville, USA, p: 1117.

18. Van Soest PV, Robertson JB, Lewis BA (1991) Methods for dietary fiber, neutral detergent fiber, and nonstarch polysaccharides in relation to animal nutrition. J Dairy Sci 74: 3583-3597.

19. Tang SX, Tayo GO, Tan ZL, Sun ZH, Shen LX, et al. (2008) Effects of yeast culture and fibrolytic enzyme supplementation on in vitro fermentation characteristics of low-quality cereal straws. J Animal Sci 86: 1164-1172.

20. Zhang CM, Guo YQ, Yuan ZP, Wu YM, Wang JK, et al. (2008) Effect of octadeca carbon fatty acids on microbial fermentation methanogenesis and microbial flora in vitro. Animal Feed Sci Technol 146: 259-269.

21. Rius AG, Kittelmann S, Macdonald KA, Waghorn GC, Janssen PH, et al. (2012) Nitrogen metabolism and rumen microbial enumeration in lactating cows with divergent residual feed intake fed high-digestibility pasture. J Dairy Sci 95: 5024-5034.

22. Jiao J, Lu Q, Tan Z, Guan L, Zhou C, et al. (2014) In vitro evaluation of effects of gut region and fiber structure on the intestinal dominant bacterial diversity and functional bacterial species. Anaerobe 28: 168-177.

23. Li M, Penner GB, HernandzSanabria E, Oba M, Guan LL (2009) Effects of sampling location and time, and host animal on assessment of bacterial diversity and fermentation parameters in the bovine rumen. J Appl Microbiol 107: 1924-1934.

24. Kittelmann S, Seedorf H, Walters WA, Clemente JC, Knight R, et al. (2013) Simultaneous amplicon sequencing to explore co-occurrence patterns of bacterial, archaeal and eukaryotic microorganisms in rumen microbial communities. Plos One 8: e47879. 
Citation: Iqbal MW, Zhang Q, Zou C, Yang Y, Huang C, et al. (2018) Subtropical Forages Differentially Influenced the Ruminal Fermentation and Microbial Community of Jersey Cow In Vitro. J Vet Sci Technol 9: 558. doi:10.4172/2157-7579.1000558

Page 8 of 8

25. Schloss PD, Westcott SL, Ryabin T, Hall JR, Hartmann M, et al. (2009) Introducing mothur: Open-Source, Platform-Independent, and community-Supported Software for Describing and Comparing Microbial Communities. Appl Environ Microbiol 75: 7537-7541.

26. McDonald IR, Bodrossy L, Chen Y, Murrell JC (2008) Molecular ecology techniques for the study of aerobic methanotrophs. Appl Environ Microbiol 74: 1305-1315.

27. Seedorf H, Kittelmann S, Henderson G, Janssen PH (2014) RIM-DB: a taxonomic framework for community structure analysis of methanogenic archaea from the rumen and other intestinal environments. Peer J 2: e494.

28. Kittelmann S, Janssen PH (2011) Characterization of rumen ciliate community composition in domestic sheep, deer, and cattle, feeding on varying diets, by means of PCR-DGGE and clone libraries. FEMS Microbiol Ecol 75: 468-481.

29. Janssen PH, Kirs M (2008) Structure of the archaeal community of the rumen. Appl Environ Microbiol 74: 3619-3625.

30. Menke KH (1988) Estimation of the energetic feed value obtained from chemical analysis and in vitro gas production using rumen fluid. Animal Res Dev 28: 7-55.

31. Rymer C, Huntington JA, Williams BA, Givens DI (2005) In vitro cumulative gas production techniques: History, methodological considerations and challenges. Animal Feed Sci Technol 123: 9-30.

32. Soto EC, Yanez-Ruiz DR, Cantalapiedra-Hijar G, Vivas A, MolinaAlcaide E (2012) Changes in ruminal microbiota due to rumen content processing and incubation in single-flow continuous-culture fermenters. Animal Prod Sci 52: 813-822.

33. Mould FL, Kliem KE, Morgan R, Mauricio RM (2005) In vitro microbial inoculum: A review of its function and properties. Animal Feed Sci Technol 123: 31-50.

34. Rodriguez R, Mota M, Castrillo C, Fondevila M (2010) In vitro rumen fermentation of the tropical grass Pennisetum purpureum and mixtures with browse legumes: Effects of tannin contents. J Animal Physiol Nutr 94: 696-705.

35. Zicarelli F, Calabro S, Cutrignelli MI, Infascelli F, Tudisco R, et al. (2011) In vitro fermentation characteristics of diets with different forage/ concentrate ratios: comparison of rumen and faecal inocula. J Sci Food Agric 91: 1213-1221.

36. Williams AG, Coleman GS (1992) The rumen protozoa. Springer, New York, USA.

37. Demeyer DI, Van Nevel CJ (1979) Effect of defaunation on the metabolism of rumen micro-organisms. British J Nutr 42: 515-524.
38. Morgavi DP, Sakurada M, Mizokami M, Tomita Y, Onodera R (1994) Effects of ruminal protozoa on cellulose degradation and the growth of an anaerobic ruminal fungus, Piromyces sp. strain OTS1, in vitro. Appl Environ Microbiol 60: 3718-3723.

39. Lengowski MB, Zuber KH, Witzig M, Mohring J, Boguhn J, et al. (2016) Changes in rumen microbial community composition during adaption to an in vitro system and the impact of different forages. Plos One 11: e0150115.

40. Saro C, Ranilla MJ, Tejido ML, Carro MD (2014) Influence of forage type in the diet of sheep on rumen microbiota and fermentation characteristics. Livest Sci 160: 52-59.

41. Huws SA, Lee MR, Muetzel SM, Scott MB, Wallace RJ, et al. (2010) Forage type and fish oil cause shifts in rumen bacterial diversity. FEMS Microbiol Ecol 73: 396-407.

42. Pope PB, Mackenzie AK, Gregor I, Smith W, Sundset MA, et al. (2012) Metagenomics of the Svalbard reindeer rumen microbiome reveals abundance of polysaccharide utilization loci. Plos One 7: e38571.

43. Li Z, Wright AD, Liu H, Bao K, Zhang T, et al. (2015) Bacterial Community Composition and Fermentation Patterns in the Rumen of Sika Deer (Cervus nippon) Fed Three Different Diets. Microbial Ecol 69: 307-318.

44. Brown VE, Rymer C, Agnew RE, Givens DI (2016) Relationship between in vitro gas production profiles of forages and in vivo rumen fermentation patterns in beef steers fed those forages. Euro J Protistol 56: 51-59.

45. Grilli DJ, Fliegerova K, Kopecny J, Lama SP, Egea V, et al. (2016) Analysis of the rumen bacterial diversity of goats during shift from forage to concentrate diet. Anaerobe 42: 17-26.

46. Jin W, Cheng YF, Mao SY, Zhu WY (2014) Discovery of a novel rumen methanogen in the anaerobic fungal culture and its distribution in the rumen as revealed by real-time PCR. BMC Microbiol 14: 104.

47. Yang L (2016) Comparative genomics of rumen methanogens. $\mathrm{PhD}$ Thesis, Massey University, Palmerston North, New Zealand.

48. Gurelli G (2016) Rumen ciliates of domestic cattle (Bos taurus taurus) in Kastamonu, Turkey, with the description of a new species. Euro J Protistol 56: 51-59.

49. Coleman GS (1992) The rate of uptake and metabolism of starch grains and cellulose particles by Entodinium species, Eudiplodinium maggii, some other entodiniomorphid protozoa and natural protozoal populations taken from the ovine rumen. J Appl Bacteriol 73: 507-513. 